thyroid is given to patients whose urine showed excess of toxins, viz., uric acid, ethereal sulphates and pigment, it either produced little or no effect, or caused fever and debility without improvement. The best results have been obtained with patients who had been for some time in the institution and whose elimination had been attended to. I would infer from the above that the physiologic action of thyroid is, 1 , increased production of lymphocytes and increased cell production generally, especially in the mucous membranes; 2 , an arrest of the destruction of the polynuclear cells.

The above hypothesis would help to account for its success in some reported cases of albuminaria.

\section{THE PALLIATIVE TREATMENT OF CANCER OF THE CERVIX UTERI AND BLAD. DER IN WOMEN.}

Read at the Thirteenth Annual Meeting of the New York State Medical Association, Oct. 14, 1896 .

BY NATHAN G. BOZEMAN, M.D.

GYNECOLOGIST TO ST. FRANCIS HOSPITAL, J. C., ST. MARY'S HOSPITAL, HOBOKEN, AND BAYONNE CITY HOSPITAL,

I shall confine my remarks to the treatment of carcinoma of the cervix uteri and the bladder in the stage of the disease when any operative measure may be of doụtful expedience, or in cases where operations have been undertaken and the disease still progresses, the condition of the patients being most distressing from hemorrhage, sepsis, incontinence of urine and all the evils resulting therefrom. My interest has been aroused in this class of patients, and no doubt you feel the same, because of their hopeless condition and the difficulty of caring for them in our public hospitals. Certainly, treatment will be directed to alleviating their suffering and rendering their surroundings as wholesome as possible.

Women afflicted with inoperable epithelioma of the cervix uteri, when they are not undergoing any treatment, complain most frequently of hemorrhage and a foul-smelling, bloody discharge from the vagina. The vaginal examination reveals a hard unyielding band of sclerous tissue in front of the cervix extending nearly around the vagina and constricting it; beyond this is the diseased cervix, which is either destroyed, leaving a deep cavity with necrotic walls, or there may be a cauliflower growth on the cervix. Usually digital examination causes excessive hemorrhage, which is controlled by placing the patient in the supported knee-elbow position, exposing the cervix by introducing a perineal elevator guided by the finger and gently brushing over the bleeding surface with a solution of persulphate of iron, or if necessary, touching it with the Paquelin cautery, then packing or columning the vagina with dry cotton. Great caution must always be observed not to injure the ulcerated surface with the end of the speculum. This accident I have found causes more bleeding than the digital examination. To guard against this $I$ also use the knee-elbow position.

In some cases the first indication is to overcome the partial stricture of the vagina which I have referred to, in order to gain free access to the disease and to allow the uninterrupted escape of the discharges. This is accomplished by means of hard rubber intravaginal dilators of suitable sizes to admit of easy introduction. They are worn for several hours a to day and are removed for douching. This gradual dilatation is continued until the contraction has yielded. The treatment sometimes is painful and has to be alternated with columning the vagina. The column of antiseptic gauze and dry cotton I employ in all cases after first dusting on aristol or some other antiseptic powder, for a twofold reason: pressure and absorption of the secretions. When it is properly introduced it may be worn forty-eight hours, then removed for thorough vaginal douching during the next twenty-four hours. It produces pressure on the diseased surface and against the constricting band in the vaginal wall. The secretions are retained in it for a considerable time and it prevents bleeding; consequently the surface of the ulcer is maintained in a fairly clean state. I treat my patients about three times a week and I find that they are kept quite free of pain and are not annoyed by the discharges. The disease has a natural tendeney to extend, but in none of my cases has a perforation occurred in to the rectum.

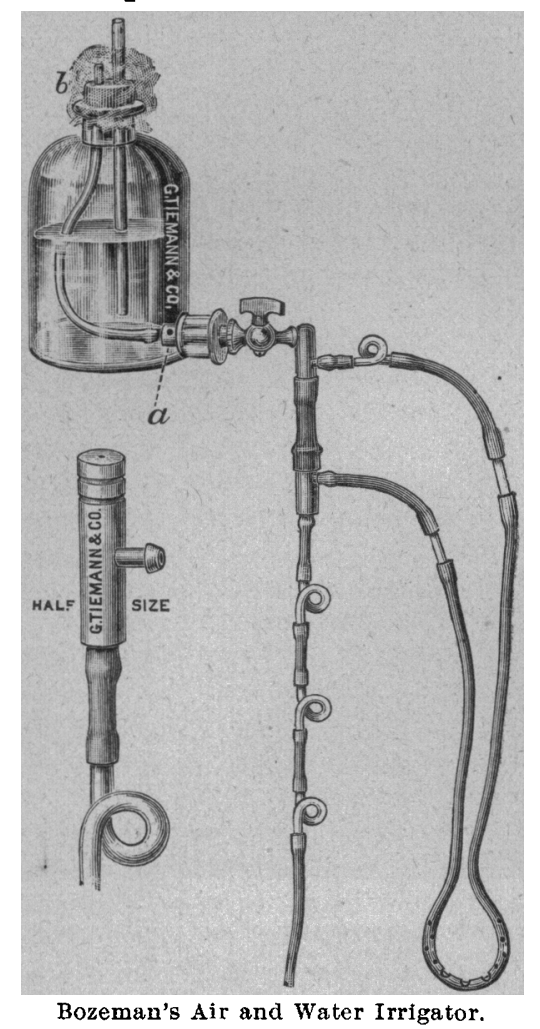

I have had several where the bladder became involved and here is when I believe most of the pain from the disease begins. The first manifestation of the extension to the bladder is a severe form of cystitis with excessive vesical tenesmus and frequent urination, but when the perforation of the vesico-vaginal septum takes place and the urine finds a free outlet, all these painful symptoms subside; a vesico-vaginal fistula is then established and the bladder has physiologic rest.

Some of us are familiar with the pitiable and loathesome condition of urinary fistula cases in former times, when the sloughing of the vaginal and bladder walls was due to prolonged pressure of the head in the parturient state, and we all know of the successes of my father in his untiring efforts to close these openings and restore such patients to health, so that now by following his teachings we are enabled to close all forms of vesico-vaginal fistulæ. Those caused by carcinomatous ulceration, however, should 
never be closed. We must content ourselves with other means of treatment.

In three cases which I have had under observation I have used continuous irrigation of the vagina and bladder, alternating with Dr. Nathan Bozeman's vesical drainage support, which is worn in the vagina and collects the urine and conduets it into a soft rubber receptacle which is attached at the knee.

Now as to the continuous irrigation. When this process is kept up for any length of time we must of course economize the fluid which we use, so I have had constructed an irrigator ${ }^{1}$ which does this by mixing air with the solution and the two, while coursing through a perforated tube in the vagina when the patient is in a recumbent or sitting posture, produces a current which carries off with it all secretions and particles of debris. The reservoir, a glass bottle, stands upon a table near the bed and is connected with the perforated tube in the vagina. The bottle has a constant outflow, that is, as the fluid escapes from it by the opening at the bottom, air passes down the long glass tube and takes its place. The pressure is thus maintained constant and it is represented by the weight of the column of fluid extending from the lower end of the perpendicular glass tube to the circular opening at the bottom of the vessel. The stopcock does not regulate the velocity of the efflux but shuts off or turns on the flow. A certain proportion of the fluid, as it escapes from the bottle, collects in a small reservoir, where it is also maintained at a constant level. In the bottom of it is a round opening, the area of which is such that with a column of liquid two inches high one-half an ounce escapes per minute. This is made to produce suction. The excess collects in an intermittent syphon and is discharged by it. The suction pump and the intermittent syphon being connected with the perforated tube in the vagina alternating columns of air and fluid pass quickly through it, carrying off with them the urine as it is secreted. Three hours is about the longest time that a bottle holding one gallon will irrigate and drain. I have now under observation a patient who has been using the irrigator with very great comfort to herself for nearly a year. In her case $I$ am also making applications once a week of hydrogen peroxid. The upper portion of the vagina, the cervix and the bladder are destroyed, but still she can be drained and is comparatively easy. In summing up I would recommend:

1. Keep the seat of the disease in an aseptic condition.

2. Prevent or counteract any narrowing of the vagina in front of the disease.

3. When the bladder becomes involved use effective irrigation and drainage to carry off the urine as soon as it is secreted.

\section{THE THERAPY OF CALCIUM SULPHID IN INFLAMMATORY DISEASES OF THE AIR PASSAGES. \\ W. BAYARD SHIELDS, M.D. \\ ST. FRANCIS, ARK.}

My attention was first attracted to the therapeutical uses of calcium sulphid in inflammatory diseases of the air passages by an article which appeared in the Journal of the American Medical Association,

1"An Air and Water Irrigator and Drain for Prolonged Douching in Deep Cavities." New York Medical Journal for May 27, 1893.
April, 1892, written by Edward F. Wells of Chicago. Dr. Wells has been using this drug in tuberculosis for a number of years and claimed to have had good results in selected cases. He had antedated Burgeon's treatment of rectal injections of sulphuretted hydrogen gas by several years. It seems that his attention had first been called to this treatment by noticing that his tubercular patients who frequented sulphur springs improved more rapidly and uniformally than those going elsewhere. There seems to be no doubt in my mind that to a certain extent that this drug has a beneficial but not curative effect in tuberculosis, as my experience leads me to know that I have seen cases in which it ameliorated the symptoms, resulting in a number of cases in an improvement in general condition and a gradual reduction of fever conducing to the general comfort and well being of the patient. In several there seemed to be a cure, but I am satisfied after a more extensive use of the drug that I was mistaken in my diagnosis, especially as there was no microscopic examination of the sputa made, although they had other signs of tubercular disease. These apparent cures were probably cases of purulent bronchitis, a disease not uncommon in very miasmatic districts and frequently accompanying remittent and intermittent malarial fever. In these fevers with the lung complication I invariably give a calcium sulphid and quinin combined with excellent results. In abscess of the lungs it seems to have a beneficial effect, liquifying the contents of abscess more rapidly and hastening its discharge.

Within the past two years I have treated not less than fifty cases of pneumonia with an extremely small per cent. of deaths, giving quinin four grains, calcium sulphid one grain every two hours for four doses and then every three hours in connection with 1 -75th of a grain of nitroglycerin, the latter in place of alcohol. Pneumonia is a disease of a serious nature in the section in which I am located on account of the complications of anemia following malarial disorders, and my death rate was much larger under the old treatment including the free use of alcohol that it has been under my present mode of treating this disease. The calcium salts seem to inhibit the pneumonic inflam. mation to a great extent, in which way I do not know, but imagine on account of it being an anti-uric acid remedy of no feeble power, and that the uric acid diathesis is a predisposing cause which favors the activity of the pneumococcus in the production of this dis. ease. Nitroglycerin, a remedy which has been advocated a number of years in pneumonia, has in my hands filled my utmost expectations in relieving the lungs by dilating the arterioles of the system and hence relieving the venous congestion. It also more than takes the place of alcohol as a stimulant as it has no bad effect on the digestion, and flushes the kidneys, thus assisting to relieve the body of effete material.

It is more especially in the inflammatory diseases of the throat, tonsillitis and quinsy, that I have most remarkable and gratifying results by the use of calcium sulphid. I had not seen the use of this drug advocated in these affections, but going upon the well-known fact that its use modified the furuncular inflammation, frequently preventing the formation of abscess, I was lead to the conclusion that it might in the same way inhibit the tonsillar inflammation. I commenced with small doses, one-fourth of a grain every three hours, but not getting good results, increased to one grain doses every hour until four doses were 